\title{
Hepcidin in malaria superinfection: can findings be translated to humans?
}

\section{To the Editor:}

With great interest we read the paper by Portugal et al. ${ }^{1}$, who reported that increased production of the iron-regulatory hormone hepcidin is responsible for inhibition of the liver-stage of malaria infection during ongoing blood-stage parasitemia.

Febrile malaria in humans is indeed associated with high circulating hepcidin levels ${ }^{2}$, but, in our opinion, two aspects of this study need further consideration. First, the authors excluded a role for immune responses in preventing malaria superinfection by using mice deficient in components of various pathways of the innate and adaptive immune systems. However, the authors did not report the circulating hepcidin concentrations or hepatic hepcidin expression levels in these mice. Their model predicts that hepatic hepcidin expression would be unchanged in these mice, yet inflammatory cytokines are key inducers of hepatic hepcidin expression ${ }^{3,4}$. Moreover, confirmation of their findings in hepcidin-knockout mice would have further strengthened their conclusion ${ }^{5}$.

Second, the limited data available in humans are not apparently consistent with the idea that an increase in hepcidin production inhibits liver-stage malaria infection. Chronic hepatitis $\mathrm{C}$ virus (HCV) infection is highly prevalent in many malaria-endemic regions, and recent evidence has shown HCV infection to be associated with reduced hepatic hepcidin expression with secondary hepatocyte iron accumulation ${ }^{6,7}$. According to the model proposed by Portugal et al. ${ }^{1}$, this would be expected to result in a favorable growth environment for liver-stage parasites. Instead, the emergence of blood-stage malaria parasites was delayed in people with $\mathrm{HCV}$ in Gabon ${ }^{8}$. Of course, other factors related to HCV infection, such as immunosuppression, may negate the effects of hepcidin on the liver-stage infection, and these clinical observations, therefore, do not fully refute a role for hepcidin.

In conclusion, Portugal et al. ${ }^{1}$ have reported intriguing data that suggest a role for hepcidin in malaria pathogenesis beyond anemia and iron dysregulation, but additional clinical studies are needed to substantiate the claims.

COMPETING FINANCIAL INTERESTS

The authors declare no competing financial interests.

Susanne van Santen ${ }^{1}$, Quirijn de Mast ${ }^{1}$, Dorine W Swinkels ${ }^{2}$ \& Andre J A M van der Ven ${ }^{1}$

${ }^{1}$ Department of General Internal Medicine, Radboud University Nijmegen Medical Centre, Nijmegen, The Netherlands. ${ }^{2}$ Department of Laboratory Medicine, Radboud University Nijmegen Medical Centre, Nijmegen, The Netherlands.

email:s.vansanten@aig.umcn.nl

1. Portugal, S. et al. Nat. Med. 17, 732-737 (2011).

2. de Mast, Q. et al. J. Infect. Dis. 199, 253-262 (2009).

3. Nemeth, E. et al. J. Clin. Invest. 113, 1271-1276 (2004).

4. Kemna, E. et al. Blood 106, 1864-1866 (2005).

5. Lesbordes-Brion, J.C. et al. Blood 108, 1402-1405 (2006).

6. Fujita, N. et al. Mol. Med. 13, 97-104 (2007).

7. Girelli, D. et al. J. Hepatol. 51, 845-852 (2009).

8. Ouwe-Missi-Oukem-Boyer, O. et al. PLoS ONE 6, e16034 (2011).
Portugal, Armitage, Newbold, Drakesmith and Mota reply:

van Santen et al. ${ }^{1}$ have commented on our recent study showing that blood-stage parasitemia in mice, above a certain threshold, impairs the growth of secondary liver-stage Plasmodium infections ${ }^{2}$. We attribute this phenomenon to hepcidin ${ }^{2}$, known to be upregulated by blood-stage parasites $^{2-5}$.

The authors suggest that experiments in hepcidin-knockout mice would reinforce our conclusion that hepcidin upregulation is involved in the observed protection against superinfection. Hepcidin is both an acute-phase protein and a homeostatic hormone. Since hepcidin is the primary controller of systemic iron homeostasis, mice deficient in hepcidin by 2 months of age have significant iron accumulation ${ }^{6}$ with clear hepatocyte iron overload and increased serum iron. Thus the baseline iron conditions in these mice are very different from those in wildtype mice. The increased liver iron may well enhance liver Plasmodium growth if it is available for pathogen uptake, as we showed by giving extra iron to mice and measuring liver parasite load ${ }^{2}$. Alternatively, iron overload is associated with generation of reactive oxygen species to which blood and liver-stage ${ }^{7}$ Plasmodium species are sensitive. Therefore, interpretation of data obtained in these mice would be difficult and would not yield a definitive conclusion.

An alternative approach would be to prevent the upregulation of hepcidin activity in wild-type mice with blood-stage infection. Neutralizing antibodies to hepcidin are one possibility for such experiments, and these are under development ${ }^{8}$ but are not currently commercially available to our knowledge. Targeting the pathways that lead to hepcidin upregulation during blood-stage malaria infection is a second possibility. However, the pathways leading to hepcidin induction during malaria remain to be elucidated. Hepcidin is typically induced during inflammation by cytokines including interleukin- 6 (IL-6) ${ }^{9-11}$, but our data suggested the bone morphogenetic protein pathway may also contribute to the upregulation of hepcidin observed during blood-stage Plasmodium infection in mice $^{2}$. We investigated hepatic hepcidin mRNA expression (Hamp1) in both MyD88-knockout and IL-6-depleted mice infected with blood-stage parasites and observed protection against superinfection in both. Hepcidin levels were also elevated compared to control mice in both cases, suggesting alternate pathways of upregulation do indeed exist during malaria infection (data not shown). These levels were above the threshold that leads to liver-stage impairment. However, in three out of nine IL-6-depleted mice, hepcidin upregulation 